\title{
OMAE2013-10896
}

\section{WAKE-INDUCED TRANSVERSE VIBRATION OF TWO INTERFERING CYLINDERS IN TANDEM ARRANGEMENT: MODELLING AND ANALYSIS}

\author{
Milad Armin, Narakorn Srinil ${ }^{*}$ \\ Department of Naval Architecture and Marine Engineering, University of Strathclyde \\ Glasgow, Scotland, UK, "corresponding author: narakorn.srinil@strath.ac.uk
}

\begin{abstract}
A considerable number of numerical and experimental studies have been performed on the problem of vortex induced vibration (VIV) of an isolated circular cylinder. A very few studies have considered a practical situation where cylinders are deployed in clusters. This study presents a mathematical fluid-structure interaction modelling and analysis of two flexibly-mounted circular cylinders arranged in tandem and subject to fluid cross flows. The hydrodynamic lift forces and their time variations are approximated by two different semiempirical wake oscillator models based on the van der Pol and Rayleigh equations. These nonlinear wake oscillators are coupled with linear structural oscillators through the acceleration and velocity coupling terms, respectively. A direct numerical time integration approach is used to predict the response amplitude behaviors and parametrically investigate the vortex- and wake-induced vibration transverse response of the two interfering upstream and downstream cylinders. Some empirical coefficients are calibrated against available, although very limited, computational fluid dynamics results. Preliminary parametric studies are conducted with the case of varying reduced flow velocity, and some insightful aspects on the effect of mass and damping ratio are highlighted. Depending on system parameters, numerical prediction results based on the van der Pol and Rayleigh equations are compared, and a combination of the two wake oscillators is suggested as a new model for predicting the vortex and wake-induced of the two interfering cylinders.
\end{abstract}

\section{INTRODUCTION}

Cylindrical structures such as marine risers subjected to vortex-induced vibrations (VIV) are of major concerns in various offshore engineering applications. If risers are located sufficiently close to each other as, for example, the development of a large hydrocarbon field requires a significant number of risers deployed in clusters, vortices generated at the wake of the upstream structure can cause a buffeting force acting on the downstream structure, leading to the sustained structural vibrations, possible clashing and subsequent structural fatigue failure. These phenomena are the well-known combined vortex- and wake-induced vibrations (VIV and WIV) of structures.

Although the understanding of the behavior of each riser is important but their interaction effect is crucial too, especially near the sea water surface where risers are often located close to each other. In this circumstance, risers may collide which may result in an unwanted dynamic scenario, e.g., leading to a reduced fatigue life of risers. Nevertheless, the prediction models relevant to multiple cylinders in cross flows are still lacking in the literature and needed from a practical viewpoint.

Early studies have focused on crosswise VIV and some models have been developed upon the idea of a wake oscillator (Bishop and Hassan, 1964). These models are amenable to a van der Pol or Rayleigh equation fulfilling a self-excited, selflimiting and nearly harmonic oscillation with finite amplitude. These nonlinear equations are coupled with the equation of motion of an elastically-supported rigid cylinder with one or two degrees of freedom (DOF). However, a much less focused research area in this field is to consider an array of multiple cylinders subjected to VIV and WIV.

Shiau and Yang (1987) considered VIV of two cylinders in tandem and proposed a Rayleigh-type equation (with a cylinder velocity coupling term) to model a fluctuating lift force due to a flowing wind. Their model, which is also considered in the present study, is based on the following, amongst others, key assumptions.

- The upstream cylinder is subject to a lift force (due to its own wake) whereas the downstream cylinder is subject to both a lift force and a buffeting force (originating from the vortices created by the front cylinder).

- The effect of downstream cylinder motion on the upstream cylinder is neglected since the two cylinders are considered to be a fair distance apart. 
More recently, Facchinetti et al. (2002) also considered a problem of VIV of two cylinders in tandem but proposed a van der Pol-type equation (with a cylinder acceleration coupling term) to model a fluctuating lift force due to a water flow. This model is also considered in the present study as an alternative model. However, as an initial investigation, no in-line motion effect, mean/fluctuating drag forces or other hydro-elastic instability is taken into account.

In this study, we aim to model and numerically predict the free vibration responses of two identical cylinders in tandem arrangement, with the use of two different wake oscillators modelling the hydrodynamic lift/buffeting forces. The goals are to make a comparison of obtained nonlinear dynamic responses of both upstream/downstream cylinders and to modify the wake oscillator models if necessary, by calibrating with some relevant computational fluid dynamics (CFD) results.

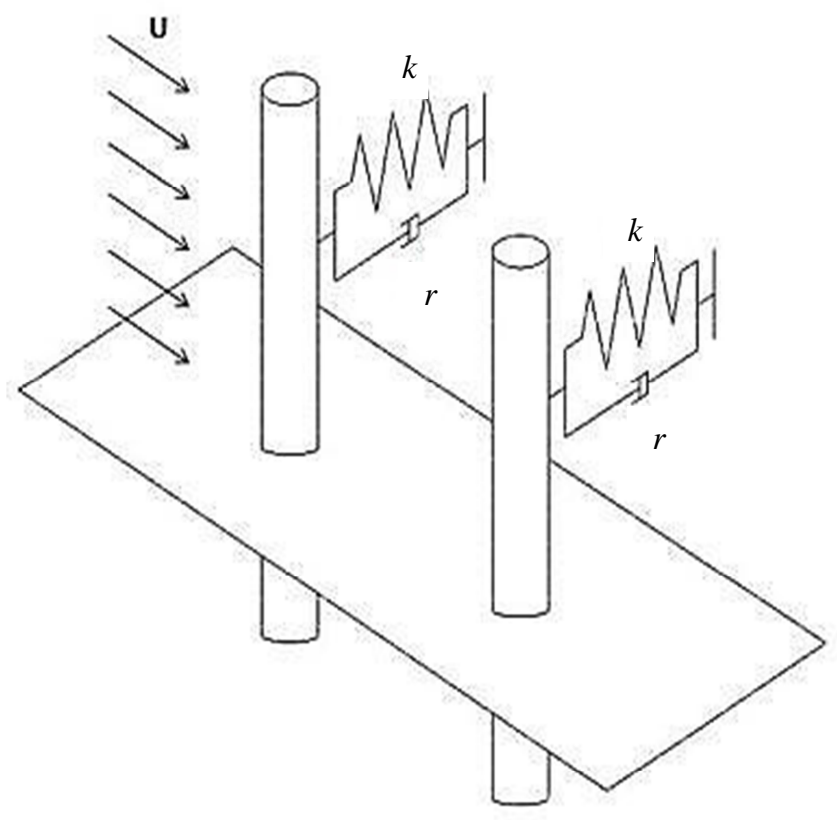

Fig. 1 Model of two oscillating cylinders in tandem

\section{MODELS OF TWO CYLINDERS IN TANDEM}

Figure 1 displays a schematic model of two elasticallysupported circular cylinders of diameter $D$, arranged in tandem, subject to a uniform two-dimensional flow and oscillating with one degree of freedom in the transverse cross-flow direction. Both cylinders are in line with a uniform flow of velocity $U$. The equation of motion of each cylinder is described by a linear spring-mass-damped oscillator as

$m \ddot{Y}+r \dot{Y}+k Y=S$

where $(\bullet)$ denotes a derivative with respect to time $T, m$ consists of structural $m_{s}$ and fluid-added mass $m_{f}$, namely $m=m_{s}+m_{f}$

$m_{f}=C_{M} \rho \frac{D^{2} \pi}{4}$

$\mu=\frac{\left(m_{s}+m_{f}\right)}{\rho D^{2}}$

$\rho$ is the fluid density, $C_{M}$ the added mass coefficient and $\mu$ the dimensionless mass ratio to be used in Eq. (13). $r$ in Eq. (1) is the linear damping coefficient including the effect of viscous damping, e.g. of the support, and the fluid-added damping,

$r=r_{s}+r_{f}$

$r_{f}=\gamma \omega \rho D^{2}$

Herein, $\omega$ is the vortex shedding frequency $\omega=2 \pi \mathrm{St} U / D$ where St is the Strouhal number, $\gamma$ a stall parameter being a function of the oscillation amplitude and mean sectional drag coefficient. In Eq. (1),

$S=\frac{1}{2} \rho U^{2} D C_{L}$

where $C_{L}$ represents the lift coefficient. By introducing the dimensionless variables,

$Y=D y$

$t=T \omega_{n}$

Eq. (1) becomes

$m \omega_{n}{ }^{2} \ddot{y}+r \omega_{n} \dot{y}+k y=S / D$

where the natural frequency $\omega_{n}=(k / m)^{1 / 2}$. By letting the damping ratio $\xi=r / 2 \omega_{n} m$, the dimensionless equation of each cylinder motion is

$\ddot{y}+2 \xi \dot{y}+y=a q$

where $q$ is the reduced vortex lift coefficient and $a$ is the mass parameter, defined by

$q=2 \frac{C_{L}}{C_{L_{0}}}$

$a=\frac{\delta^{2}}{2} \frac{C_{L_{0}}}{8 \pi^{2} \mathrm{St}^{2} \mu}$

where $C_{L 0}$ is the lift coefficient of the stationary cylinder taken as 0.3 (Hartlen and Currie, 1970). Note that Eq. (11) is widely considered in the literature. Nevertheless, some studies have recently considered nonlinear structural oscillators and found the potential effect of geometric coupling associated with cubic nonlinearities (Srinil and Zanganeh, 2012). 


\section{Rayleigh Wake Oscillator}

A Rayleigh-type nonlinear oscillator model with a negative nonlinear damping term has been suggested by Hartlen and Currie (1970) to describe the wake of an oscillating cylinder. This oscillator, coupled with the velocity of the vibrating cylinder, reads

$\ddot{q}-\varepsilon \delta \dot{q}+\frac{\gamma{C_{L_{0}}}^{2}}{4 \delta}(\dot{q})^{3}+\delta^{2} q=\frac{2 A}{C_{L_{0}}} \dot{y}$

in which $\varepsilon$ and $A$ are empirical constants to be tuned with available experiment data, and

$\delta=\frac{\omega_{s}}{\omega_{n}}$

$\gamma=\frac{4 \varepsilon}{3 C_{L_{0}}^{2}}$

For a single cylinder undergoing VIV, Eqs. (11) and (15) can be solved for $y$ and $q$. In the case of two cylinders in tandem, similar systems of equations of motion for $\left(y_{1}, y_{2}\right)$ and $\left(q_{1}, q_{2}\right)$ are considered for both circular cylinders. However, as previously mentioned, the downstream cylinder is subject to both lift and buffeting forces, the former as a result of its own wake whereas the latter as a result of the vortex wake from the front cylinder.

Therefore, due to this combined effect of VIV and WIV, the equation of rear cylinder motion may be assumed, following Shiau and Yang (1987) which accounted for the time delay $t_{1}$ (i.e. the time required for the vortices generated by the upstream cylinder motion to reach the downstream cylinder) effect, as

$\ddot{y}+2 \xi \dot{y}+y=a\left[q_{2}(t)+q_{1}\left(t+t_{1}\right)\right]$

$t_{1}=\frac{2 \pi}{\delta} \times \frac{d}{D} \times \frac{D}{l}$

where $d$ is the centre-to-centre distance between the two cylinders, $l$ is the vortex spacing, $d / D$ is the cylinder spacing ratio. The reduced flow velocity parameter - which is varied in the parametric study - may be related to Eq. (19) via

$V_{r}=\delta / S t$

Finally, the system of dimensionless equations describing the two cylinder displacements and the two wake variables read

$$
\left\{\begin{array}{c}
\ddot{y}_{1}+2 \xi \dot{y}_{1}+y_{1}=a q_{1} \\
\ddot{y}_{2}+2 \xi \dot{y}_{2}+y_{2}=a\left[q_{2}(t)+q_{1}\left(t+t_{1}\right)\right] \\
\ddot{q}_{1}-\varepsilon \delta \dot{q}_{1}+\frac{\gamma C_{L_{0}}{ }^{2}}{4 \delta}\left(\dot{q}_{1}\right)^{3}+\delta^{2} q_{1}=\frac{2 A}{C_{L_{0}}} \dot{y}_{1} \\
\ddot{q}_{2}-\varepsilon \delta \dot{q}_{2}+\frac{\gamma C_{L_{0}}{ }^{2}}{4 \delta}\left(\dot{q}_{2}\right)^{3}+\delta^{2} q_{2}=\frac{2 A}{C_{L_{0}}} \dot{y}_{2}
\end{array}\right.
$$

\section{van der Pol Wake Oscillator}

Alternatively, the fluctuating characteristic of the vortices may be modeled by the van der Pol equation. Following Facchinetti et al. (2004), the wake oscillator may be written as

$\ddot{q}+\varepsilon \delta\left(q^{2}-1\right) \dot{q}+\delta^{2} q=A \ddot{y}$

where the coupling term in the right-hand side of above equation is proportional to the cylinder acceleration, $\varepsilon$ and $A$ are empirical coefficients as in Eq. (15). Thus, the system equations governing the two in-tandem cylinders are as follows

$$
\left\{\begin{array}{c}
\ddot{y_{1}}+2 \xi \dot{y}_{1}+y_{1}=a q_{1} \\
\ddot{y_{2}}+2 \xi \dot{y}_{2}+y_{2}=a\left[q_{2}(t)+q_{1}\left(t+t_{1}\right)\right] \\
\ddot{q}_{1}+\varepsilon \delta\left(q_{1}^{2}-1\right) \dot{q}_{1}+\delta^{2} q_{1}=A \ddot{y}_{1} \\
\ddot{q}_{2}+\varepsilon \delta\left(q_{2}^{2}-1\right) \dot{q}_{2}+\delta^{2} q_{2}=A \ddot{y_{2}}
\end{array}\right.
$$

Depending on the wake oscillator used, system Eq. (21) or (23) is directly solved using a numerical time integration routine to obtain unknowns steady amplitudes of both cylinders $\left(y_{1}, y_{2}\right)$ and wakes $\left(q_{1}, q_{2}\right)$. The case of increasingly varying flow velocity is considered. Variables of $q_{1}$ (behaving as a wake-induced or buffeting force) and $q_{2}$ (behaving as a lift force) are coupled through the oscillator of the downstream cylinder. This consideration is different from the work of Facchinetti et al. (2002) where $q_{1}$ and $q_{2}$ are coupled through the van der Pol wake oscillator of the downstream cylinder with an additional coupling term but without a time delay variable.

Herein, the specific aim is to determine the ability of each model to capture the overall response amplitude diagram when compared to published results via computational fluid dynamics (CFD) or experimental study. As an initial study, CFD results from the recent work of Prasanth and Mittal (2009) are considered. Therein, two cylinders in tandem (with the mass ratio $m^{*}=10$, damping ratio $\xi=0$, and spacing ratio $d / D=5.5$ ) were allowed to oscillate in both streamwise and transverse directions, in contrast to the present study whose in-line motion is assumed to be negligible. In this study, we use the same $m^{*}$, $\xi$ and $d / D$ as above values with $l / D=4.25$ (Sallet 1969).

\section{PARAMETRIC INVESTIGATION}

There are two empirical coefficients $\mathcal{E}$ and $A$ governing the VIV and WIV responses of both cylinders, and their appropriate values have to be calibrated versus the referenced CFD results, based on the (i) Rayleigh (Eq. 21) or (ii) van der Pol (Eq. 23) oscillator. To reduce the calibration tasks, in this study $\mathcal{E}$ and $A$ are assumed to be the same for the two wake oscillators controlling $q_{1}$ and $q_{2}$ of each model.

Figures 2 and 3 show the effect of varying $A$ and $\varepsilon$ on upstream $\left(Y_{1} / D\right.$ or $\left.y_{1}\right)$ and downstream $\left(Y_{2} / D\right.$ or $\left.y_{2}\right)$ amplitude responses, respectively, with the latter also displaying the corresponding CFD results. With fixed $\varepsilon=0.3, Y_{1} / D$ and $Y_{2} / D-$ and associated excitation ranges - increase with increasing $A$ (Fig. 2). This holds also for the van der Pol model (similar results are thus not shown herein). 

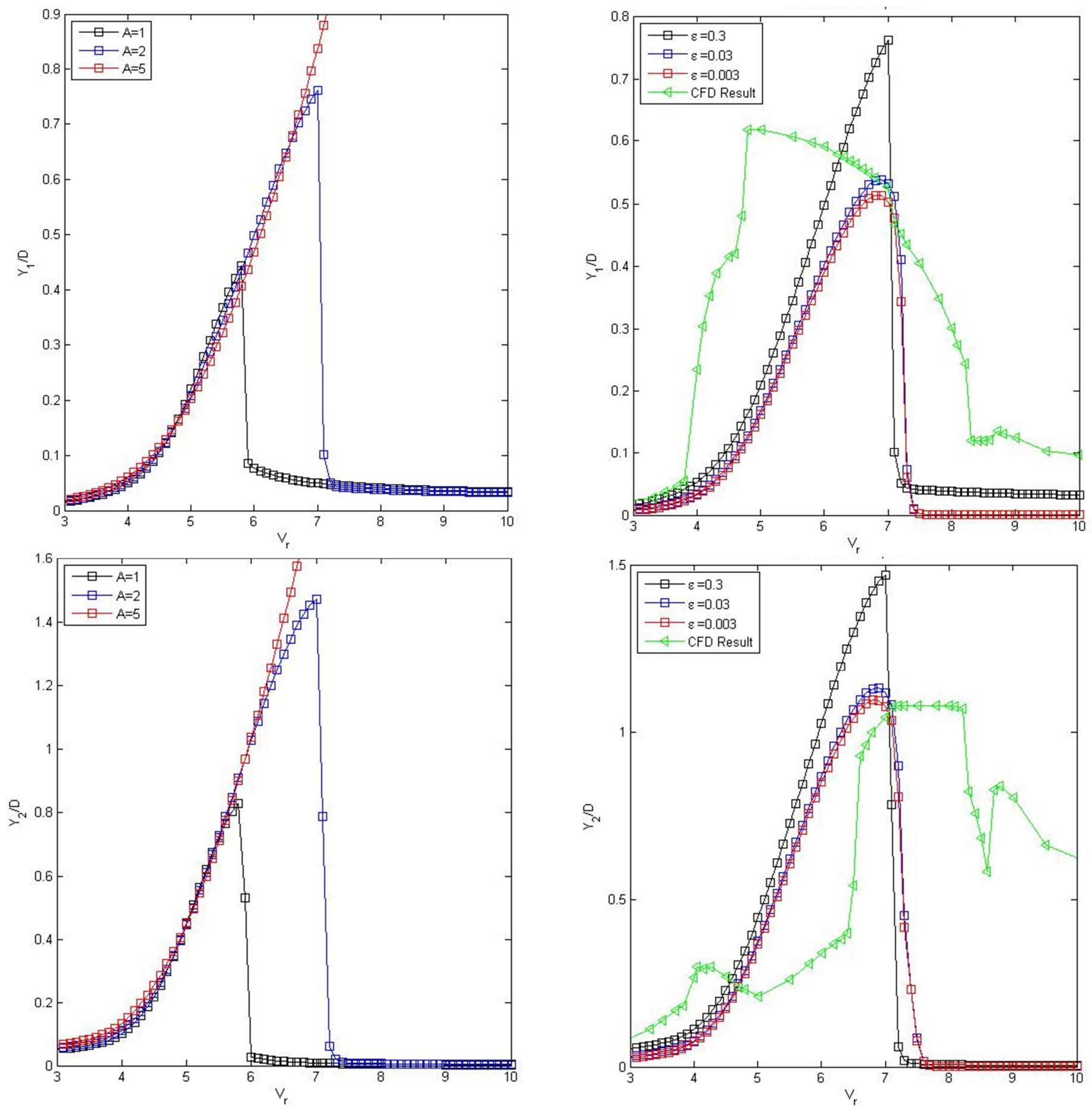

Fig. 2 Cylinder responses with Rayleigh model: varied $A$

The $A=2$ case seems to provide a reasonable amplitude results with maximum $Y_{1} / D$ and $Y_{2} / D$ being equal to about 0.75 and 1.50 , respectively. Next, $A=2$ is fixed and $\varepsilon$ is varied (Fig. 3 ); it is seen that $Y_{1} / D$ and $Y_{2} / D$ increase - although the excitation range slightly changes - with increasing $\varepsilon$. The case with $A=2$ and $\varepsilon=0.003$ is chosen for the Rayleigh model.

Fig. 3 Cylinder responses with Rayleigh model: varied $\varepsilon$

For the van der Pol model, the effect of varying $\varepsilon$ is exemplified in Fig. 4 for a fixed $A=12$. Contrary to Fig. 3, $Y_{1} / D$ and $Y_{2} / D$ in Fig. 4 increase with decreasing $\varepsilon$. This is the main difference in results obtained between the Rayleigh and van der Pol models, perhaps due to the structural coupling terms in the wake oscillators being the velocity or acceleration. 

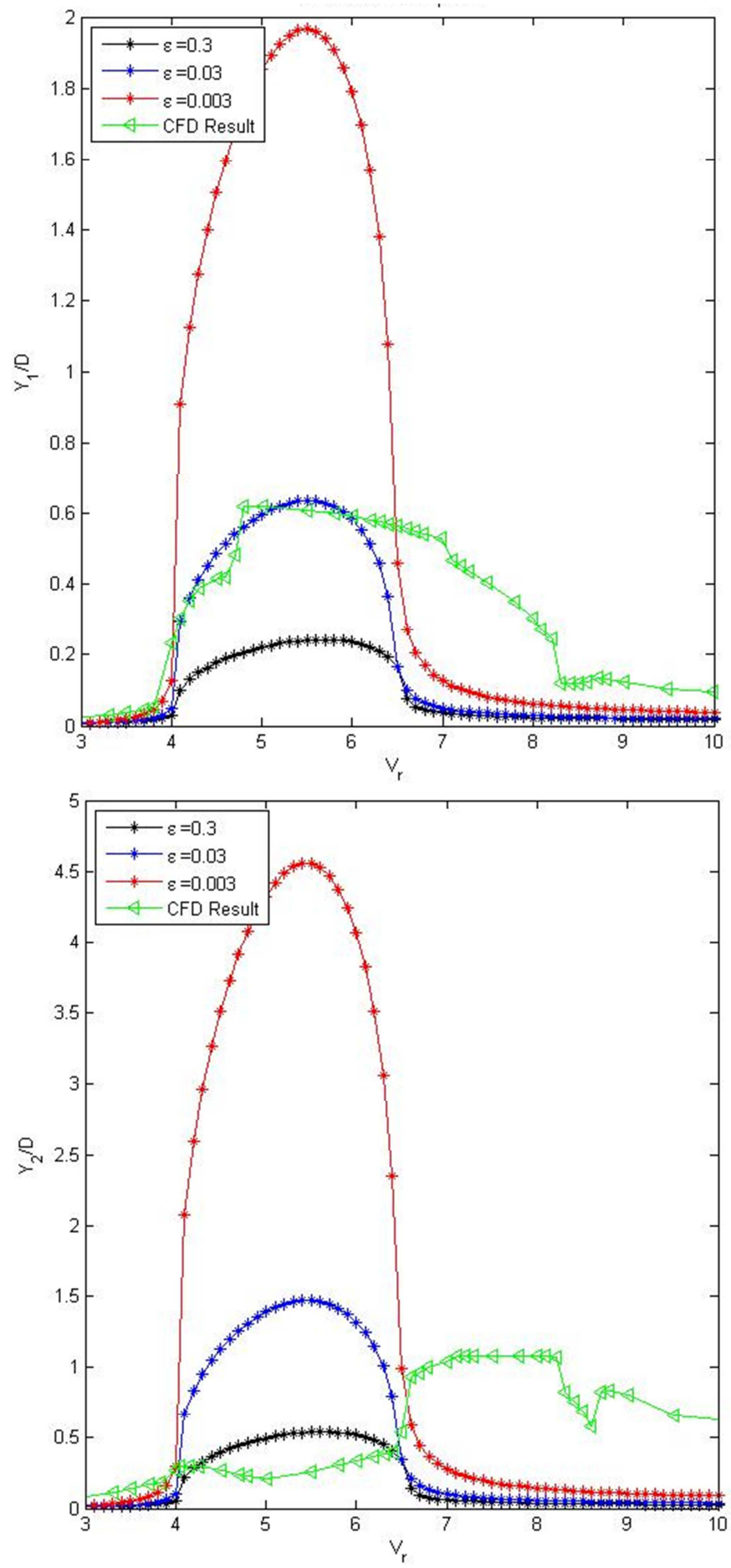

Fig. 4 Cylinder responses with van der Pol model: varied $\varepsilon$

The most suitable case for the van der Pol oscillators seems to be that with $\varepsilon=0.03$ and $A=12(\varepsilon=0.3$ and $A=12$ in Facchinetti et al. 2004 for a single cylinder), in terms of maximum amplitudes, although discrepancies between numerical prediction and CFD results of the downstream cylinder - in terms of the excitation range - are quite large.
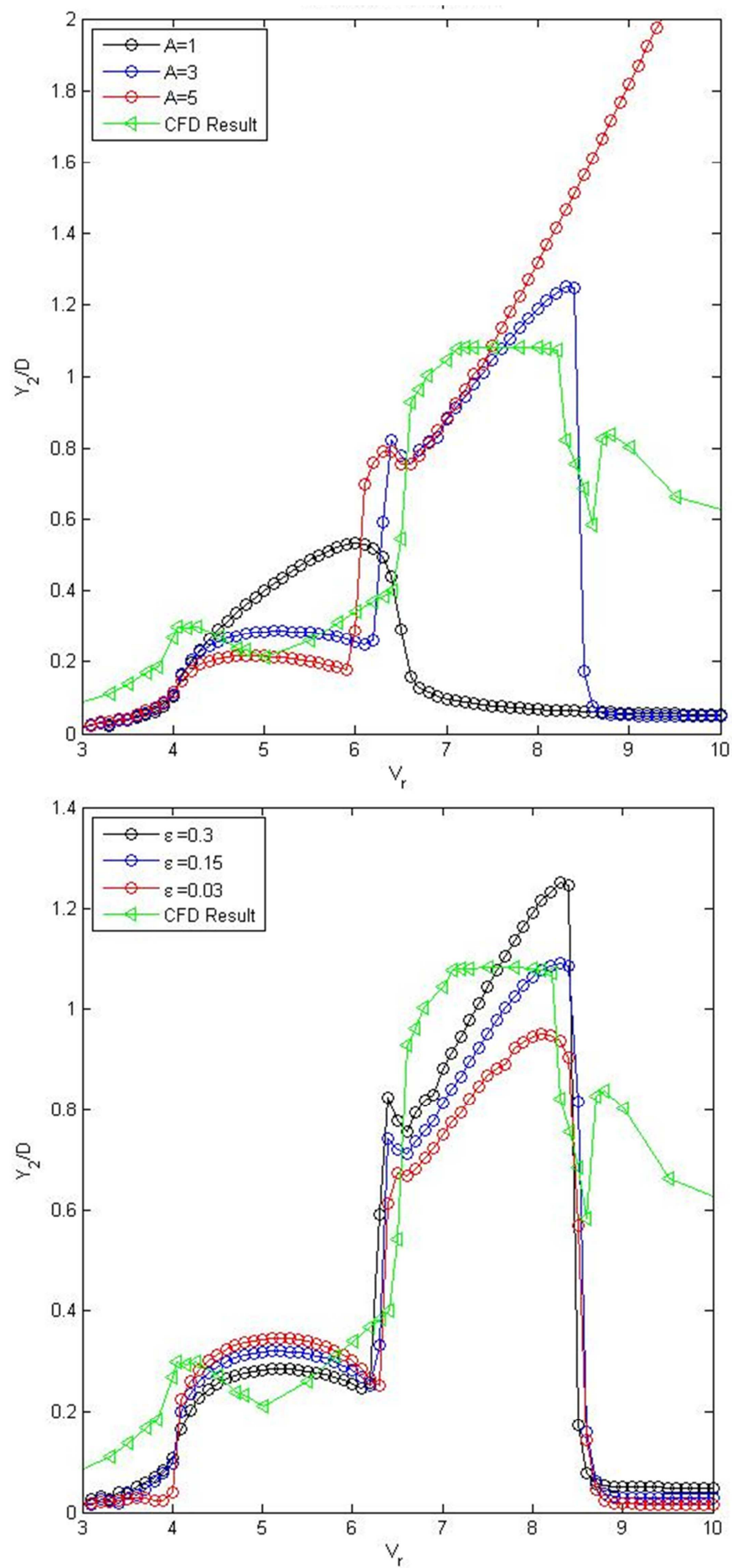

Fig. 5 Downstream cylinder responses with combined model: varied $A(\varepsilon=0.3)$ and $\varepsilon(A=3)$

To further improve the prediction of downstream cylinder response, a combined model with van der Pol $\left(q_{1}\right)$ and Rayleigh $\left(q_{2}\right)$ oscillators are now proposed as a new alternative model. Only the coefficients $\varepsilon$ and $A$ governing $q_{2}$ are now tuned (i.e. fixed $\varepsilon=0.03$ and $A=12$ for $q_{1}$ ), and amplitude response 

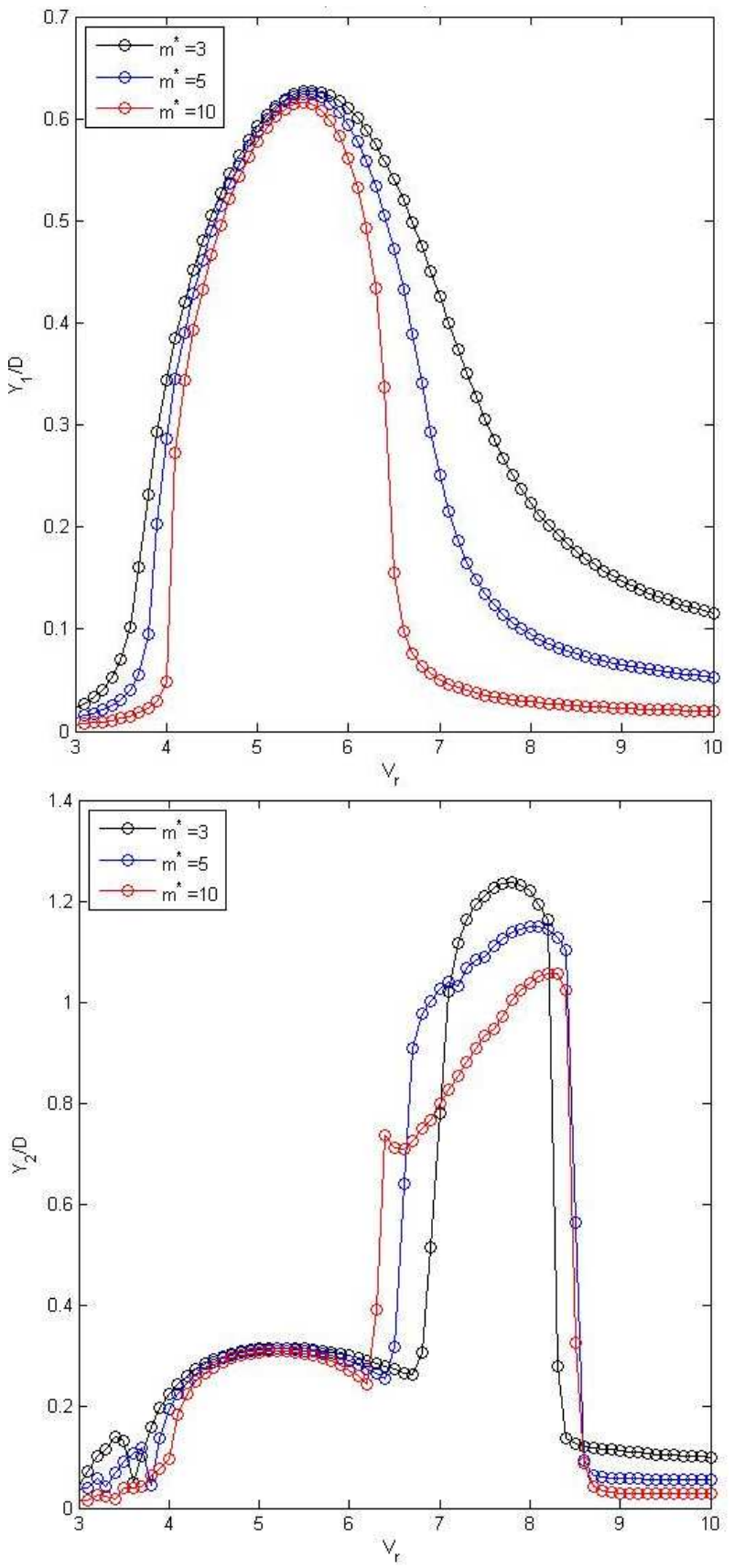

Fig. 6 Effect of mass ratio on two in-tandem cylinder responses predicted by the combined model $(\xi=0.001)$

results of the downstream cylinder with varying $A$ and $\varepsilon$ are shown in Fig. 5. It can be seen that results with the specified $\varepsilon$ $=0.15$ and $A=3$ for $q_{2}$ provide the best fits to CFD results. Interestingly, this new combined model captures the multi-peak feature of the downstream cylinder seen in the CFD study in which maximum amplitudes occur in the approximate range of $4<V_{r}<6$ and $7<V_{r}<9$. However, there is a sharp drop in the numerically-predicted response at $V_{r} \approx 9$ while the CFD response persists at higher $V_{r}$.
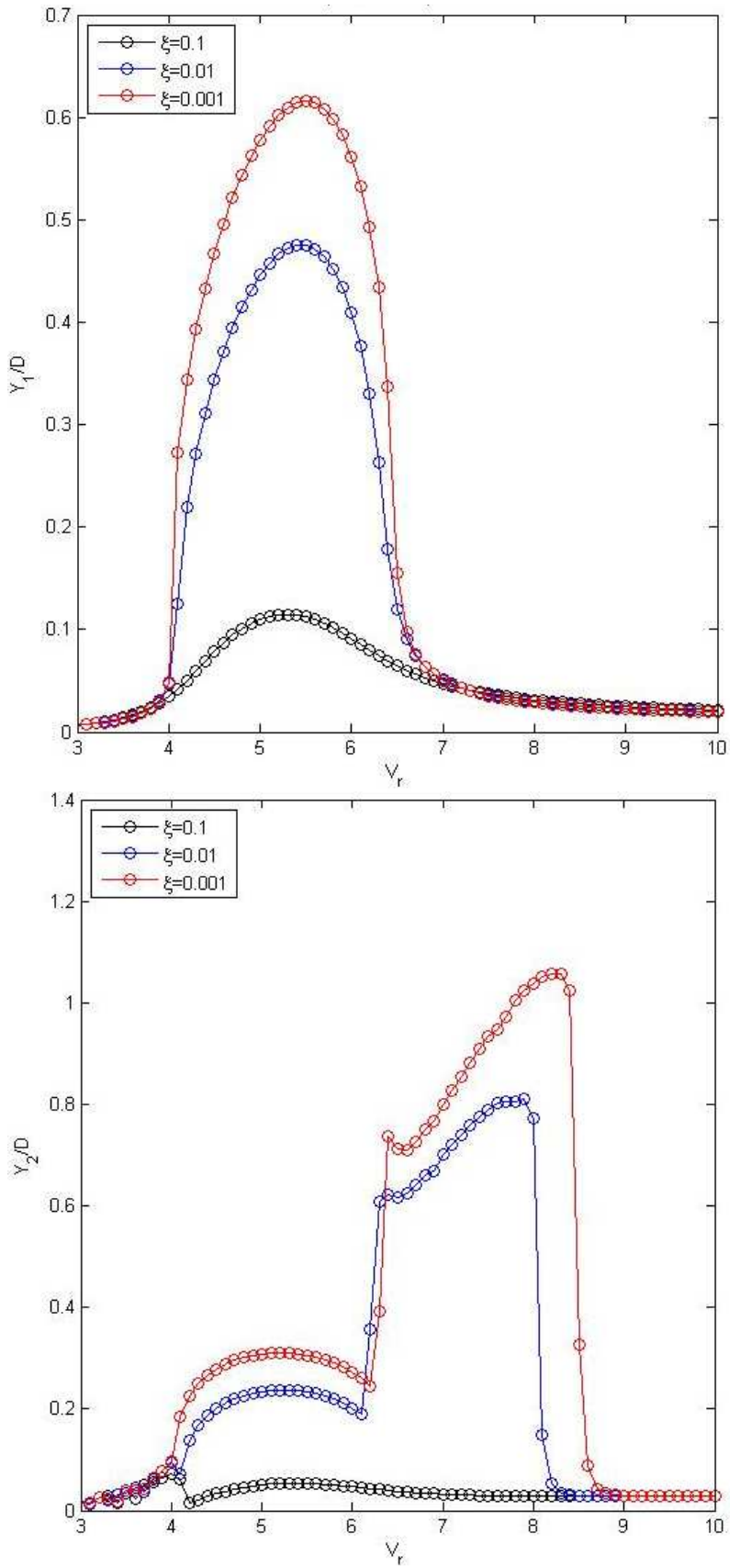

Fig. 7 Effect of damping ratio on two in-tandem cylinder responses predicted by the combined model $(m *=5)$

Table 1 Summary of tuned empirical coefficients based on calibration with CFD results

\begin{tabular}{|r|r|r|c|}
\hline \multicolumn{1}{|c|}{ Model } & \multicolumn{1}{|c|}{$\boldsymbol{A}$} & \multicolumn{1}{c|}{$\boldsymbol{\varepsilon}$} & $\boldsymbol{q}_{\mathbf{1}}$ or $\boldsymbol{q}_{\mathbf{2}}$ \\
\hline Rayleigh & 2 & 0.003 & both \\
\hline van der Pol & 12 & 0.03 & both \\
\hline Combined van der Pol \& & 12 & 0.03 & $\boldsymbol{q}_{\mathbf{1}}$ \\
Rayleigh & 3 & 0.15 & $\boldsymbol{q}_{\mathbf{2}}$ \\
\hline
\end{tabular}


Table 1 summarizes and compares overall tuned empirical coefficients for each wake oscillator model used in this study.

To show the capability of the combined model, the effect of varying $m^{*}$ and $\xi$ on both upstream and downstream cylinder cross-flow responses are now illustrated in Figs. 6 and 7, respectively. It can be seen in Fig. 6 that the variation of $m^{*}$ has an influence on the lock-in range of the upstream cylinder - as in the case of single cylinder (Khalak and Williamson, 1999) whereas it has an influence on the peak, mainly associated with the second excitation range, of the downstream cylinder. In the latter case, amplitudes increase with decreasing $m^{*}$. When decreasing $\xi$ as in Fig. 7 , both $Y_{1} / D$ and $Y_{2} / D$ increase - as expected - while maintaining the associated lock-in ranges. For a high $\xi$ value, the downstream cylinder response may ultimately disappear or become negligible.

Overall, maximum attainable $Y_{2} / D$ values are nearly twice $Y_{1} / D$, and the associated excitation ranges for the upstream and downstream cylinders are different: there is a shift to a higher $V_{r}$ range for the downstream cylinder. These observations, along with the multi-peak feature of $Y_{2} / D$ response, provide a good qualitative agreement with CFD results of Prasanth and Mittal (2009). Nevertheless, more calibration with experimental results (e.g. Assi et al. 2006) and parametric studies are needed to understand such other important effects as spacing ratio and in-line oscillations.

\section{CONCLUSIONS}

A mathematical fluid-structure interaction modelling and analysis of two flexibly-mounted circular cylinders arranged in tandem and subject to fluid cross flows has been presented and investigated. The hydrodynamic lift and buffeting forces and their time variations are approximated by two different semiempirical wake oscillator models based on the van der Pol and Rayleigh equations. These nonlinear wake oscillators are coupled with linear structural oscillators through the acceleration and velocity coupling terms, respectively.

A direct numerical time integration approach is used to predict the response amplitude behaviors and parametrically investigate the vortex- and wake-induced vibration transverse response of the two interfering upstream and downstream cylinders. Some empirical coefficients are calibrated against CFD results. Preliminary parametric studies are conducted with the case of varying reduced flow velocity, and some insightful aspects on the effect of mass and damping ratio are highlighted. Depending on system parameters, numerical prediction results based on the van der Pol and Rayleigh equations are compared, and a combination of the two wake oscillators is suggested as a new model for predicting the vortex and wake-induced of the two interfering cylinders. The new model is seen to capture a multi-peak response and a multi-excitation region of the downstream cylinder observed from the CFD study.

More analytical, numerical and experimental studies are needed to improve the model terms and empirical coefficients and capture realistically various aspects of in-tandem cylinders subject to VIV and WIV.

\section{ACKNOWLEDGEMENTS}

The authors are grateful to the "Faculty Engineering Technology Studentship" given by the Faculty of Engineering, University of Strathclyde.

\section{REFERENCES}

Assi, G.R.S, Meneghini, J.A.P., Bearman, P., Casaprima, E., 2006. Experimental investigation of flow-induced vibration interference between two circular cylinders. Journal of Fluids and Structures 22, 819-827.

Bishop, R.E.D., Hassan, A.Y., 1964. The lift and drag forces on a circular cylinder oscillating in a flowing fluid. Proceedings of the Royal Society of London, Series A 277, $51-75$.

Blevins, R.D., 1990. Flow-Induced Vibrations. Van Nostrand Reinhold, New York.

Facchinetti M.L., de Lngre E., Biolly F., 2004. Coupling of structure and wake oscillators in vortex-induced vibrations. Journal of Fluids and Structures 19, 123-140.

Facchinetti, M.L., de Langre, E., Fontaine, E., Bonnet, P.A., Etienne, S., Biolley, F., 2002. VIV of two cylinders in tandem arrangement: analytical and numerical modeling. In: Proceedings of the 12th International Offshore and Polar Engineering Conference, Kitakyushu, Japan, 524-531.

Hartlen, R.T., Currie, I.G., 1970. Lift-oscillator model of vortex-induced vibration. Journal of the Engineering Mechanics Division EM5, 577-591.

Khalak, A., Williamson, C.H.K., 1999. Motions, forces and mode transitions in vortex-induced vibrations at low massdamping. Journal of Fluids and Structures 13, 813-851.

Prasanth, T. K., Mittal, S., 2009. Flow-induced oscillation of two circular cylinders in tandem arrangement at low Re. Journal of Fluids and Structures, 25, 1029-1048.

Sallet, D. W., (1969). On the spacing of Karman vortices, Journal of Applied Mechanics, 36, 370-372.

Shiau, L. Yang, T.Y., 1987. Two-cylinder model for wind vortex-induced vibration. Journal of Engineering Mechanics $113,780-789$

Srinil, N., Zanganeh, H., 2012. Modelling of coupled cross-flow/in-line vortex-induced vibrations using double Duffing and van der Pol oscillators. Ocean Engineering 53, 8397. 